\title{
Triplet Gestation- Prevention, Risks, \& Management Dilemmas
}

\author{
Blumenfeld Zeev ${ }^{*}, 1$, William Abdallah ${ }^{1}$, Sela-Guttmann Onit ${ }^{1}$ and Brook Olga Rachel ${ }^{1,2}$ \\ ${ }^{1}$ Reproductive Endocrinology \& Infertility, Department of Obstetrics and Gynecology, Rambam Medical Center, \\ Technion Faculty of Medicine, Haifa, 31096, Israel \\ ${ }^{2}$ Medical Imaging DeparOtment, Rambam Medical Center, Haifa, 31096, Israel
}

\begin{abstract}
The increased popularity of assisted reproductive technology [ART] has been associated with a significant increase in the incidence of multiple pregnancies. Indeed, $43.5 \%$ of triplet and higher order multiple births in the US were generated by ART, $38.5 \%$ were attributed to ovulation-inducing drugs without ART, and $18 \%$ occurred spontaneously. The escalating frequencies of multiple gestations, combined with the inevitable increased risks for adverse perinatal outcomes, have a significant influence on the overall measures of neonates' and infants' health and well being. In addition, multifetal pregnancies are associated with increased risks of maternal complications such as preeclampsia and other hypertensive disorders, cesarean delivery, postpartum hemorrhage, anemia, diabetes, premature rupture of membranes, placental abruption, fatty liver, and maternal death. Three maternal preconception characteristics are associated with improved outcome of triplets: multiparous, older, and tall women fare better than nulliparous, younger, and shorter controls, respectively. Whereas many obstetricians recommend on iatrogenic multifetal reduction, it may increase the risk of miscarriage, premature rupture of membranes and adverse psychological effects. In triplet gestation where the couple, is reluctant to undergo this procedure for moral or religious reasons, or due to the fear of losing the long awaited pregnancy, the recent information whereby taller patients are at a significantly lower risk of very premature delivery as compared to shorter patients, may be helpful.
\end{abstract}

Keywords: Triplets, Vanishing twins, Multifetal pregnancy reduction, Premature delivery, Multiple gestation.

\section{INTRODUCTION}

Over the past decade, there has been a significant and dramatic increase in the incidence of multiple pregnancies and triplets in most developed countries, generated by the increased popularity of ART, IVF/ET and ovulation induction [1]. Since many women, in the last decades deferred child bearing until an older age, when the fertility decreases, the necessity of ART and fertility treatments has substantially increased in these women $[1,2]$. Moreover, about one third of triplet gestations are attributed to the rising maternal age at spontaneous conception. [3]. For example, -in the two decades between 1980-2001 in the United States the number of twin deliveries high-rocketed $77 \%$ and other multiple births were tremendously increased 400- $459 \%[2,4,5]$. The overall rate of multiple deliveries in the developed countries is very high , about $3 \%-4 \%$, which is $3-4$ times higher than the natural rate prior to the era of assisted reproductive technologies (ART), or the rate in developing countries where ART is seldom or infrequently used [6]. About $43 \%$ of triplet and higher order multiple births in the USA were the result of ART, including in vitro fertilization (IVF) and gamete intrafallopian transfer (GIFT), 39\% were generated by invivo ovulation induction without ART, and $18 \%$ occurred spontaneously [7].

\footnotetext{
*Address correspondence to this author at the Reproductive Endocrinology $\&$ Infertility, Department of Obstetrics and Gynecology, Rambam Medical Center, Technion Faculty of Medicine, Haifa, 31096, Israel,

E-mail: z_blumenfeld@ rambam.health.gov.il
}

The rates of singleton, twin, triplet, and quadruplet deliveries for IVF and ICSI were $73.6 \%, 24.4 \%, 2.0 \%$ and $0.04 \%$ respectively. These rates sum up to a total multiple delivery rates of $26.44 \%$. The range of triplet deliveries generated by IVF and ICSI ranged from $0.3 \%$ to $7.0 \%$ between various European countries [8]. Twin and triplet pregnancies have been reported to have a natural incidence of $1 / 80$ for twins and 1/6,400 - 1/9,520 for triplets [9].

The total multiple delivery rate after IVF and ICSI remained practically unchanged during the last 4 years, with an optimistic tendency towards decrease in high order multiple gestations attributed most recently to the transfer of only one or two embrya [8].

The increased incidence of multiples in the general population is generated by a $20 \%$ to $30 \%$ incidence of multiple births after ART [10].

Gestational age-adjusted comparisons of outcome between singletons and multiples demonstrated conflicting results and conclusions [5]. Comparisons that corrected for relevant confounding variables have demonstrated that twins and singletons may not necessarily differ in the risks of perinatal morbidity and mortality [5].

The escalating frequencies of multiple gestations, combined with the inevitable increased risks for adverse perinatal outcomes, have a significant influence on the overall measures of neonates' and infants' health and well being [6].

Triplet gestation can arise from as few as one to as many as three ovulated and fertilized ova, in-vivo, or the replacement of one to three embryos in an IVF/ET cycle [4]. Minor 
trauma to the blastocyst during the assisted reproductive technology may possibly lead to the increased incidence of mono-zygotic twinning observed in pregnancies conceived in this manner [4].

In spontaneously conceived triplets, the incidence of bichorionicity was $44 \%$. The bi-chorionic triamniotic triplets have a 5.5-fold higher risk of adverse perinatal outcome predominantly because of the twin to twin transfusion syndrome and premature rupture of the membranes [3]. The overall prevalence of monochorionic pairs in spontaneously conceived triplet pregnancies is approximately $44 \%$, which is 2.2 times higher than the reported prevalence for twin pregnancies [3].

The frequency of monochorionic twins in a naturally conceived triplet pregnancy is approximately 50\%- $60 \%$ (Fig. 1). In contrast, investigators who assessed chorionicity in triplet gestations by ultrasound scans found that only $3 \%$ $19 \%$ of these pregnancies had monochorionic pairs. Only $5 \%$ to $10 \%$ of multiple pregnancies that are generated by ART have monochorionic pairs [3].

In humans, as the number of fetuses per pregnancy increases, the percentage of male conceptuses decreases.[4]. Stranskov \& co-workers (1946) found $51.6 \%$ males in $31 \times 10^{6}$ singleton births in USA, for twins it was $50.9 \%$, for triplets- $49.5 \%$, and for quadruplets- $46.5 \%$. In late twinning events an even higher ratio of female to male was found (75\% of monochorionic-monoamniotic and conjoined twins were female) [4].

Although triplets are disadvantaged in almost every perinatal aspect compared with singletons and twins, there may be subsets that are at a particularly high risk. The early identification of at-risk triplet pregnancies may possibly improve their outcome [3].

A paradoxical relationship between maternal age and perinatal outcomes in multiple pregnancies has been observed [11]. Triplet neonates born to older mothers fare better than triplets born to younger parturients. It is important to note, however, an exception; in subgroups known to be less likely to conceive using ART (such as in parturients belonging to a low socioeconomic class), the risk of very preterm birth, very low birth weight, and perinatal mortality increased in parallel to increasing maternal age [6].

It is well known that most triplet gestations in older women were generated by ovulation induction drugs in fertility treatments, and these women were generally of higher socioeconomic class [11]. Women who conceive through the use of ART are financially more secure, and, as a result, may have an improved health care coverage and work less during pregnancy, enjoy an improved healthcare, and their newborns usually receive a high standard of care [6].

Older women are usually of higher gravidity, which merely reflects a longer fertile period [11].

The effect of parity on triplets' birth weight suggests that the uterine milieu of the nulliparous woman is less efficient in accommodating triplets than that of the multiparous patient, but the responsible physiologic mechanism is still unknown [6].

Multiple pregnancies are regarded as serious complications of ART. The mean gestational age at birth is inversely correlated to the number of fetuses. The mean gestational

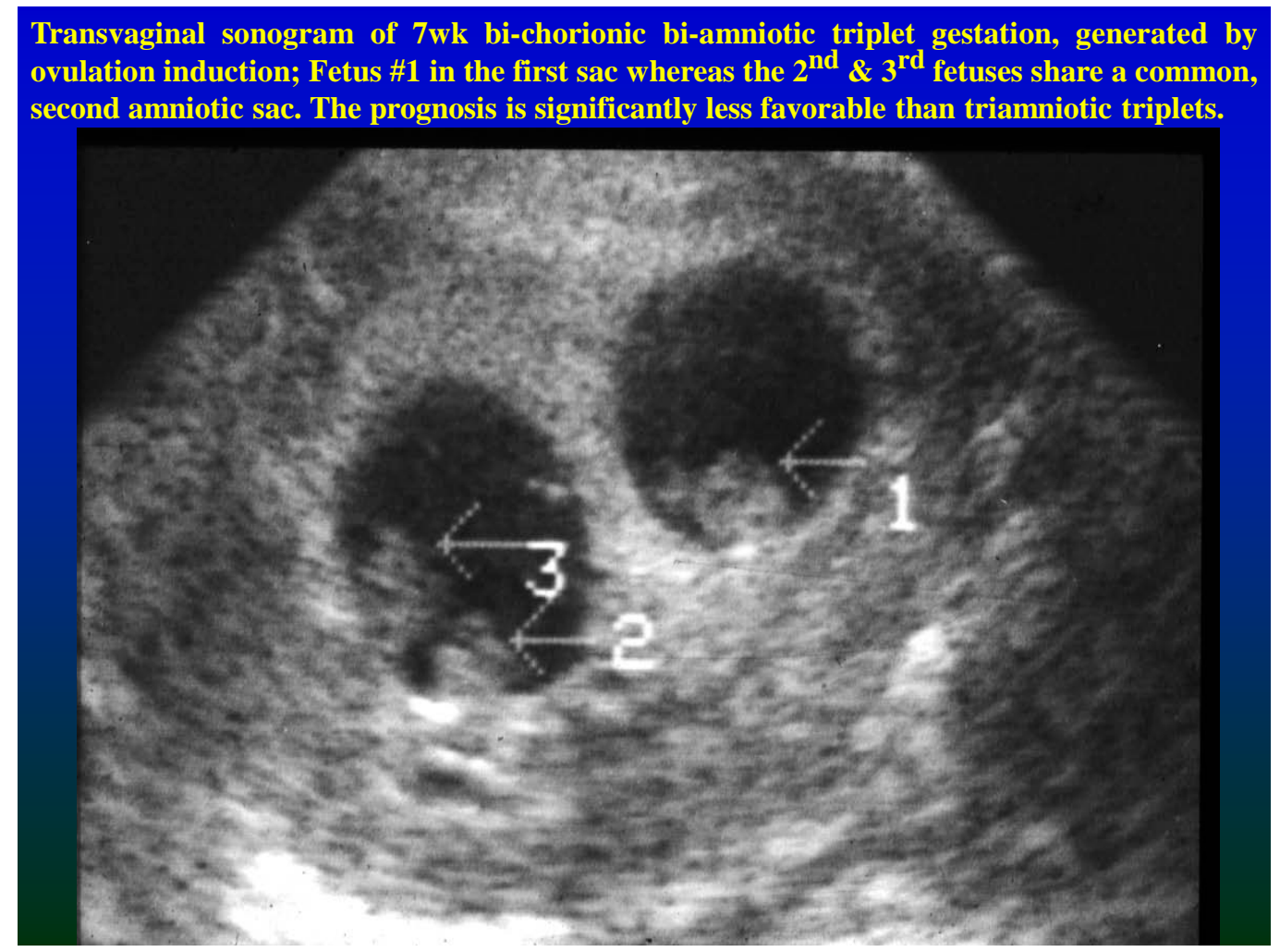

Fig. (1). A transvaginal sonogram showing a bi-chorionic bi-amniotic [BCBA] triplet gestation, seven weeks, generated by ovulation induction; One fetus is in the first sac whereas the second and third fetuses share a common, second amniotic sac. The prognosis and fetal outcome of such gestations are significantly less favorable than triamniotic triplet pregnancies. 
age for singletons is 39 weeks, for twins 35.8 weeks, and for triplets - 32.5 weeks [5].

Multifetal pregnancies are associated with both an increased risk of maternal complications as well as a high prevalence of perinatal and neonatal morbidity and mortality due to prematurity [9].

Intrauterine growth in multiples is similar to that seen in singletons until approximately 22 weeks' gestation, but afterwards there is an apparent divergence which becomes much more evident after the 28 week of gestation [5].

\section{MATERNAL ADAPTATION}

Generally, the degree of maternal physiological changes usually detected during gestation is intensified with multiple fetuses compared to those occurring with a singleton gestation [4]. Starting in the first trimester, women with multiple gestations often complain of nausea and vomiting much more than those characterizing singleton pregnancies [4].

The physiologic maternal blood volume expansion is also exaggerated in multiple gestations. The red cell mass increase is intensified as well. Subsequently, the mean blood loss during delivery is higher than in singleton birth. There is an increase in iron and folic acid requirements predisposing to a greater risk of maternal anemia [4]. A greater increase in cardiac output is also detected in multiple pregnancies as compared to singletons [4].

It is also possible that older mothers may have a higher body mass index or different height; two established factors associated with improved birth weights in multiple pregnancies [11].

Taller mothers delivered significantly heavier triplets than shorter parturients with triplet gestations [11].

The adjusted parity may not be enough to explain the potential influence of gravidity [11].

Older mothers of triplets were of higher gravidity than younger controls matched for parity [11].

There is new evidence from multiple gestations suggesting an outcome paradox: - that advanced maternal age may be associated with better outcomes [11, 12].

Zhang et al. [13] presented four potential explanations including the lower occurrence of monozygotic sets among iatrogenic conceptions, use of young donor eggs for older mothers, better antenatal care, and better financial background of the older parturient $[11,12]$.

Older patients are likely to be more "mature." Many may have finished or be at a solid midpoint in their career aspirations and have the ability or desire to devote the requested nine months exclusively to pregnancy requirements. Older mothers, especially those who conceived as a result of assisted reproduction, also are likely to have had sufficient time to increase their awareness of the special needs of the unborn fetus. This also means that these patients are more likely to seek better and more competent obstetric advice early in the first trimester. Because older women may have a higher body mass index [BMI] and be as weight conscious as their younger counterparts, they may be more likely to eat wisely, obtain a balanced diet, decrease alcohol consumption, and avoid smoking. Such potential advantages could be associated with a greater likelihood of positive outcomes [12].

Younger patients with triplets were found to be at a disadvantage compared with their older counterparts in terms of birth weight characteristics.

Currently, there is no an unequivocal and universally accepted explanation for the advantageous outcomes among triplet births in the older gravida. One potential explanation is that prior pregnancy experience influences later outcomes. According to Dickinson [14] the nulliparous uterus varies in length from 3.2 to $8.1 \mathrm{~cm}$, whereas the parous uterus varies from 5.7 to $9.4 \mathrm{~cm}$. More importantly, the weight of the nulliparous uterus is $63.2 \mathrm{~g}$ compared with $125 \mathrm{~g}$ in the multiparous counterpart [12].

A larger number of women aged 40 or older had a history of abortion compared with the younger control group (aged 25-29). Due to one or more previous abortions or miscarriages, older patients may have been more likely to possess "remodeled" uteri that were more able to withstand the rigors of distention as the pregnancy progressed [12]. The increased uterine weight associated with higher gravidity, as shown by Langlois [15], suggests that even pregnancies that end in abortions or miscarriages result in cell proliferation, which ultimately may affect the uterine performance. These findings support the contention that the uterus, unlike the ovary, does not lose its ability to function in older age [12].

It is also possible that prior cell proliferation from the remodeling process may assist in better nourishing the fetuses of older women, just as labor in the multipara is more efficient. This could explain the higher total birth weights of triplets born to older mothers compared with younger mothers [12].

A preconception maternal profile could estimate the likelihood of adverse outcomes and be used for consulting patients at risk of having or carrying a triplet gestation [6].

Three maternal pregravid characteristics are associated with improved outcome of triplets. Specifically, multiparas, older, and tall mothers fare better than nulliparas, younger, or shorter controls, respectively [6].

Elster et al. [16] have documented the effect of maternal stature on fetal size in triplets - There is positive correlation between stature and mean total triplet birth weight but not with gestational age at birth in nulliparous women with a normal pregravid BMI [6].

A particular maternal phenotype - the large mother may be evolutionarily selected to bear twins and possibly also triplets [6].

\section{MATERNAL COMPLICATIONS}

The various causes of stress in pregnancy and the likelihood of serious maternal complications are almost invariably increased with multiple fetuses as compared to singleton gestations. This should be taken into account, especially when counseling the women whose health is compromised and whose multifetal gestation is recognized early [4].

Fertility and pregnancy after the age of forty is associated with clearly demonstrable and even unique risks such as decreased fecundity, spontaneous abortion, genetic abnormali- 
ties, medical complications, fetal growth abnormalities, dysfunctional labor, higher rate of cesarean section, and maternal and perinatal morbidity and mortality [12].

Maternal complications that are more frequently encountered in multiple pregnancies [10]:

1. Hypertensive disorders:

a. Preeclamptic toxemia[PET] (HELLP syndrome, acute fatty liver)

b. Pregnancy-induced hypertension

c. Chronic hypertension

d. Eclampsia and PET

2. Anemia

3. Gestational diabetes

4. Premature uterine contractions [PUC] and premature delivery

5. Tocolytics associated complications

6. Delivery-associated complications:

a. Caesarean section

b. Operative delivery

c. Premature rupture of membranes

d. Postpartum endometritis

e. Placental abruption

Maternal complications for women carrying high order multiple gestations include increased incidence of preeclampsia, cesarean delivery, and postpartum hemorrhage, fatty liver and maternal death $[13,10]$. Higher rates of anemia, diabetes, premature rupture of membranes and other hypertensive disorders are also more commonly observed in multifetal gestations [2].

Although maternal morbidity was increased in older gravidas, the overall neonatal outcome was not affected [12].

Women with multifetal gestations were more vulnerable to starvation ketosis after fasting compared with patients bearing singleton pregnancies [4].

Mild and severe pregnancy-related hypertensive disorder increases progressively with advancing fetal number from singleton to triplets [2]. The incidence of hypertensive conditions is greater in twin, triplet, and quadruplet pregnancies
$(12.7 \%, 20.0 \%$, and $19.6 \%$, respectively) as compared with singletons (5-6.5\%, $P<0.001$ for all comparisons) [2]. Triplet gestations have a 3 -fold increase in the incidence of pregnancy-related hypertensive disease [2].

The incidence of severe pregnancy-related hypertensive conditions including severe PET, hypertension with severe proteinuria, hypertension with the development of thrombocytopenia, HELLP syndrome, renal failure, or disseminated intravascular coagulation, or development of eclampsia are significantly higher in triplet gestations as compared to singletons [2]. The rate of severe gestational hypertension was estimated to be three fold higher for twins and six-fold higher for triplets than for singleton controls [2].

The need for early delivery related to hypertension was greater with increasing fetal number through triplet gestations. Higher fetal number, nulliparity, and advanced maternal age were each independently associated with the development of pregnancy-related hypertensive disorders [2].

\section{PSYCHOLOGICAL EFFECT \& ASSISTANCE}

A multiple pregnancy is usually more stressful both physically and emotionally as compared to a singleton pregnancy because of increased body discomfort, the need for frequent monitoring, more frequent obstetric interventions, and mainly due to the association with suboptimal or unfavorable neonatal outcome [10]. Patients who conceived after ART may associate preterm birth with guilt and stress and not infrequently blame themselves and feel responsible for the suboptimal outcome [10].

The risk of stillbirth and neonatal death in twins is more than two and five times higher, respectively, than for singletons [10]. In triplets these unfortunate statistics are even higher.

A special situation with strong psychological influence is the great paradox of multifetal pregnancy reduction in couples who were desperately trying to conceive and are after finally "successful" but are "obliged" to consider termination of some embryos to allow the others to survive. The fear that all fetuses would be lost after such an intervention cannot be dismissed [10].

The procedure of multifetal reduction may possibly bring about adverse psychological effects such as grief or depression [1]. Jewish orthodox religious couples would not agree to multifetal reduction without the Rabbinical declaration

Table 1. Maternal Morbidity in Multiple Gestations Versus Singleton Pregnancy

\begin{tabular}{|c|c|c|c|}
\hline Maternal Complication & Singleton Pregnancy & Multiple Gestation & Odd Ratio \\
\hline \hline Preeclampsia \& Hypertensive Disorders of pregnancy & $6 \%$ & $13 \%$ & 2.2 \\
\hline Placental abruption & $0.8 \%$ & $2 \%$ & 2.5 \\
\hline Urinary Tract Infections & $6.7 \%$ & $8.7 \%$ & 2 \\
\hline Anemia & $4 \%$ & $22 \%-39 \%$ & $7-13$ \\
\hline Gestational Diabetes & $3 \%$ & $<1 \%$ & 0.2 \\
\hline Post-term gestation & $4 \%$ & & 2 \\
\hline
\end{tabular}


that one of the triplets or quadruplets is defined as "RODEFF" meaning possessing a potential threat, risk or even potential "killer" or " murderer" of his brother twins.

A study by McKinney et al. on the psychological effects of multifetal pregnancy reduction concluded that when the outcome of the pregnancy is successful, the reduction does not put the women in significant psychiatric symptoms [17]. Unfortunately, not infrequently the outcome of triplet gestations is less than optimal ... possibly associated with psychological or psychiatric problems.

\section{MULTIFETAL PREGNANCY REDUCTION}

Higher order multiple gestations confer significant risks to both mother and fetuses and reduce the chances of both a live birth and birth of neonates without significant handicap. Some consider the only effective treatment is a selective reduction procedure, which also increases the risk of losing the entire pregnancy.

This procedure can be performed as soon as the ultrasound records triplets with positive fetal heart rate but because of spontaneous loss and the ability to measure the nuchal translucency in each fetus and rule out other anomalies by ultrasonography as early as 11-13 weeks, according to some sonographers, the procedure of fetal reduction is usually carried out by transabdominal approach between the $12^{\text {th }}$ and $14^{\text {th }}$ week of gestation by intracardiac fetal injection of potassium chloride.
In addition higher-order multiple gestations are financially costly and expensive, not only to health insurance providers but also to the patient during and after delivery, due to necessity, in most cases to retire work and employment [4].

As the number of fetuses increases, the duration of gestation and birth weight decrease. It thus appears reasonable to assume that selective fetal reduction will improve the mean gestational age and mean birth weight in twins selectively reduced from triplets as compared with nonreduced triplets, thus decreasing the risk of prematurity. However, this procedure is not without risks including the possible loss of the entire long waited pregnancy in the previously infertile couple [9], a complication occurring in about 5-9\% of the MFPR [18].

Not infrequently it may be difficult to precisely locate the exact position of the multiple fetuses, which is important for the multifetal pregnancy reduction and essential for the selective termination [4].

Selective reduction of triplets to twins before 12 weeks results in a growth pattern typical of twins rather than triplets $[4,19]$.

The American College of Obstetricians and Gynecologists [ACOG] declared that it was still unclear and remained to be determined whether reduction of triplet gestations to twins improved the long-term outcome [6,20].

Table 2. Fetal Complications in Multiple Gestations Versus Singleton Pregnancy

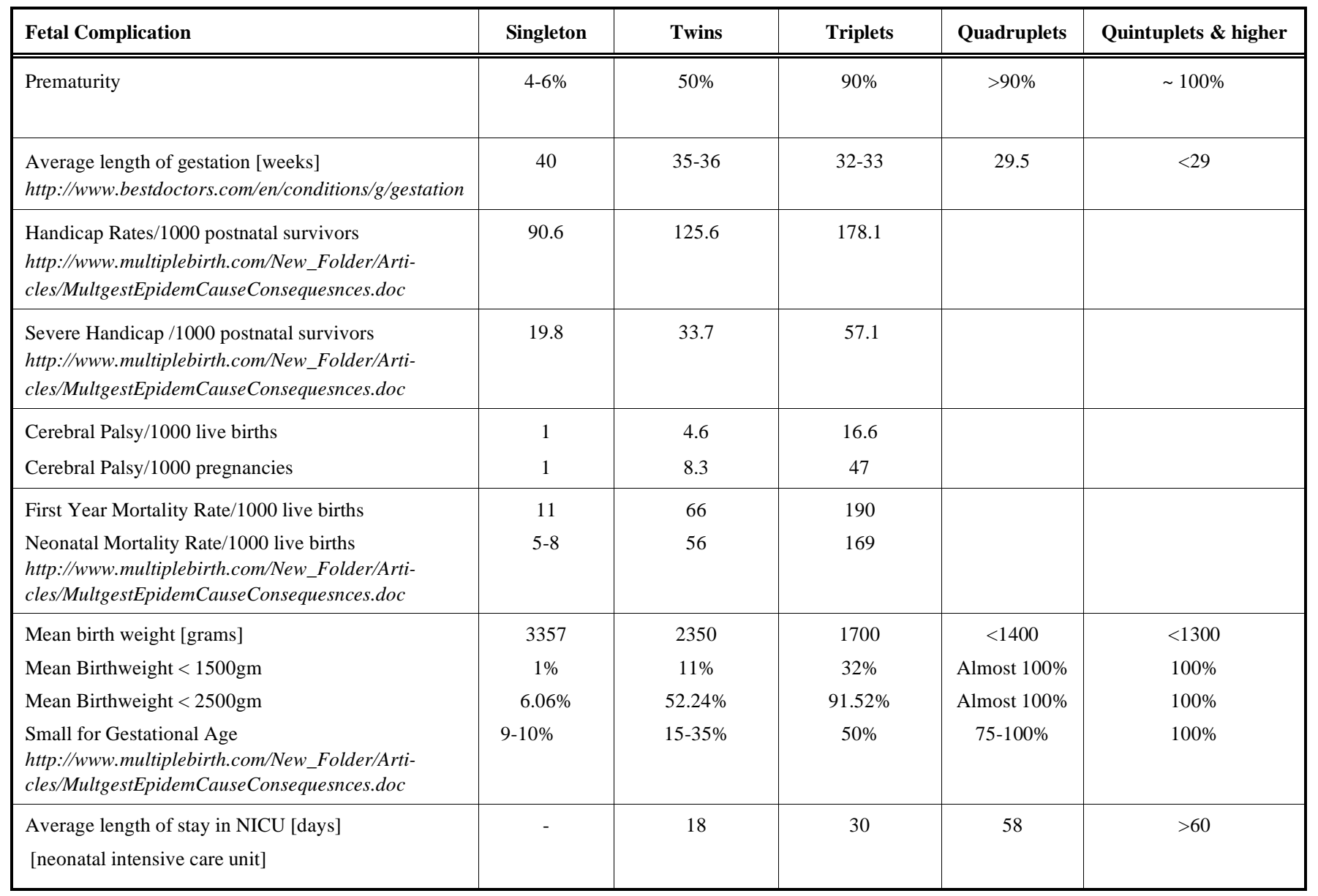


A retrospective review by Leondires et al. [21] on the outcomes of expectant management versus multifetal pregnancy reduction of 127 pregnancies suggested that multifetal pregnancy reduction does not have a significant impact on the probability of live birth or on gestational age at delivery for women with triplets who conceived via ART. According to this retrospective study and because the perinatal and neonatal improvement, along with the moral, ethical and psychological side effects of fetal reduction, these investigators suggest that couples with triplets should be counseled to make a decision based on personal choice and not on expectation of improved outcomes of the reduced triplet gestation [21].

Many obstetricians recommend that triplet gestation should undergo iatrogenic fetal reduction to twins in the second trimester, after waiting for spontaneous reductions ("vanished twin" phenomenon) to occur, usually during the first trimester [1]. However, occasionally the "vanished twin" phenomenon has occurred even at 17 weeks or even later.

The fetal reduction procedure may increase the risk of miscarriage, premature rupture of membranes and may possibly bring about adverse psychological effects and dilemmas as mentioned before $[1,10]$.

In cases of triplet gestation where the couple, in spite of the explanations and recommendations of the health-care providers, is reluctant to undergo this procedure for moral or religious reasons or due to the fear of losing a long awaited pregnancy, sometimes after longstanding infertility and expensive infertility treatments, the information generated by the studies of Blickstein et al. and Blumenfeld et al. [1, 6], may be helpful. The taller patient $(>165 \mathrm{~cm})$ may be at a significantly lower risk of very low birth weight neonates and very premature delivery as compared to the shorter patient $(<165 \mathrm{~cm})$.

\section{SPONTANEOUS PREGNANCY REDUCTION (SPR) - THE "VANISHED TWINS PHENOMENON":}

Spontaneous abortion is more likely with multiple fetuses, especially monochorionic [3].

First-trimester SPR can be recognized in $>25 \%$ of multiple pregnancies diagnosed in the early first trimester. SPR is not associated with decreased gestational age at delivery, reduced birth weight or increased incidence of pregnancyinduced hypertension [22] as documented by a prospective analysis on 709 multiple and 5962 singleton pregnancies conceived at an infertility clinic [7]. Spontaneous reduction of one or more gestational sacs and or embryos occurred before the $12^{\text {th }}$ week of gestation in $36 \%$ of twin $(95 \% \mathrm{Cl}$, $32 \%-40 \%), 53 \%$ of triplet $(95 \% \mathrm{Cl}, 44 \%-61 \%)$, and $65 \%$ of quadruplet $(95 \% \mathrm{Cl}, 46 \%-85 \%)$ pregnancies [7]. Reduction was less frequent after ovulation induction than after spontaneous ovulation [7]. In general, pregnancy duration and birth weight were inversely related to the initial gestational sac number irrespective of the final birth number [7]. More than $50 \%$ of the patients with three or more gestational sacs had spontaneous reduction before 12 weeks. The surviving fetuses weighted less and were born earlier than unreduced pregnancies with the same initial number of fetuses [7].
Multifetal pregnancy reduction (MFPR) from quadruplet and higher order gestations to twin gestations significantly decreases the risk of delivery before 28 weeks and associated neonatal and developmental morbidity, as well as maternal risks and morbidity [7].

After MFPR, triplet gestations that were reduced to twins delivered at a more advanced gestational age and had higher birth weights than unreduced triplets $[7,19,23]$.

However, other [7, 24] studies have noticed that after MFPR, triplets gestations reduced to twins delivered earlier and had lower birth weights than unreduced twins. Again, other studies [7, 19] found no differences in the length of gestation [7]. There was a trend toward decreased birth weight as the number of initial gestational sacs increased for spontaneously reduced pregnancies, compared with unreduced gestations [7].

In 2000, Leondires et al. [7,21] reported a $13.6 \%$ incidence of spontaneous reduction after the ninth week for 81 triplet pregnancies resulting from ART, but they did not stratify the results by maternal age. Lipitz et al. [23] reported the miscarriage of all embryos between the $9^{\text {th }}$ and $20^{\text {th }}$ gestational week in $13.2 \%$ of 106 expectantly managed triplets in a prospective study, but did not report how often spontaneous reduction to twins or singletons occurred [7].

After three fetal heartbeats were detected, spontaneous reduction occurred in $10 \%$ of pregnancies for patients younger than 30 years and $55 \%$ for patients 30 years and older [7].

The findings of this study clearly points out that spontaneous reduction of multifetal gestations is a common occurrence in multiple pregnancies, and it suggests that the decision to perform MFPR does not need to be finalized until late first trimester or early second trimester. Obstetricians managing pregnancies in which there were initially 3 or more GSs, which later reduced spontaneously to singleton or twin gestations, should be aware that such pregnancies may deliver 4 to 10 days earlier with neonates weighting $119 \mathrm{~g}$ to $429 \mathrm{~g}$ less than unreduced singletons and twins [7].

\section{FETAL COMPLICATIONS}

Multifetal gestations are at an increased risk for spontaneous abortion, congenital malformations, and monozygosity-related conditions such as twin to twin transfusion syndrome, growth aberrations, reduced birth weight, preterm birth and prematurity. The incidence of maternal and fetal morbidity and mortality increases concomitantly with the number of fetuses in utero $[2,4,5,10]$.

The incidence of congenital malformations is significantly increased in twins and higher-order multiple gestations as compared to singleton pregnancies [4]. This increase is almost entirely due to the high incidence of structural defects in monozygotic twins [4]. According to Schinzel and associates [25], the anomalies in monozygotic twins generally fall into one of three categories:

1. Defects resulting from twinning itself, which may be considered to be a teratogenic event by some investigators. This category includes conjoined twins, acardiac anomaly, syrenomelia, neural-tube defects, and holoprosencephaly [4, 25]. 
2. Defects resulting from vascular interchange between monochorionic twins. Vascular anastomoses can give rise to reverse flow with acardia in one twin. Alternatively, if one twin undergoes fetal demise and intravascular coagulation develops, these connections can allow emboli to reach the living twin. Vascular anastomoses may also conduct dramatic blood pressure fluctuations, causing defects such as microcephaly, hydranencephaly, intestinal atresia, aplasia cutis, and limb amputation $[4,25]$.

3. Defects that occur as a result of fetal crowding. Examples include talipes equinovarus (clubfoot) and/or congenital hip dislocation. Dizygotic twins and triplets are also subject to these abnormalities $[4,25]$.

Multifetal gestations are more likely to be characterized by low birthweight than singleton pregnancies due mostly to restricted fetal growth and preterm delivery.

More than two thirds of triplet pregnancies are complicated by fetal growth restriction [4] which is the main etiology responsible for the increased incidence of low birth weight in multifetal gestations [1]. The triplets growth curve is therefore different from the respective curve for singletons.

Size discordance can result from unequal placentation or from umbilical cord abnormalities (such as: velamentous insertion of the umbilical cord, marginal insertion, vasa previa) or from different genetic fetal growth potential including genetic syndromes and fetal malformations and relative placental insufficiency [4].

Pregnancy and perinatal outcomes with relation to maternal age showed improved outcomes in older parturients with triplets. Data about older mothers of quadruplets and quintuplets also documented favorable survival outcomes with increasing maternal age [12].

The National Center for Health Statistics Matched Multiple Data Set was used further to compare neonatal death rates in complete triplet sets by maternal age [12]. In triplets, the neonatal death rates declined from 54.3 per 1000 in parturients aged 25 to 29 to 21.5 per 1000 in mothers aged 40 or older. Regarding twins, the decline was not as dramatic but was still impressive (20.5 per 1000 in young mothers aged 25-29 versus 13.2 per 1000 in parturients aged 40 or older) (A. Branum, unpublished data, 2002) [12].

A retrospective study by Adegbite et al. [3] that determined the perinatal outcome of spontaneously conceived triplet pregnancies in relation to chorionicity found that the overall perinatal mortality rate was 151.5 per 1000 total births, with bichorionic triamniotic triplets having a 5.5-fold higher risk than trichorionic triamniotic gestations The bichorionic triamniotic [BCTA] triplets have a higher risk of premature delivery before 30 weeks of gestation and birth weight of $<1000 \mathrm{~g}$ and higher risk of PROM than those of trichorionic triamniotic pregnancies (Figs. 1, 2). In this study [3], $30 \%$ were delivered before 30 weeks of gestation, with bichorionic triplets having a 4.6 -fold higher risk. This difference may be attributed to four times higher prevalence of preterm and premature rupture of the membranes [PPROM]. Although the cause for this observation is unclear, it may be due to either polyhydramnios or an increase in the total amniotic fluid volume [3].

Intrauterine demise of the compromised fetus may induce neurologic damage of the remaining normal co-fetus through feto-fetal transfusion by the communicating placental ves-

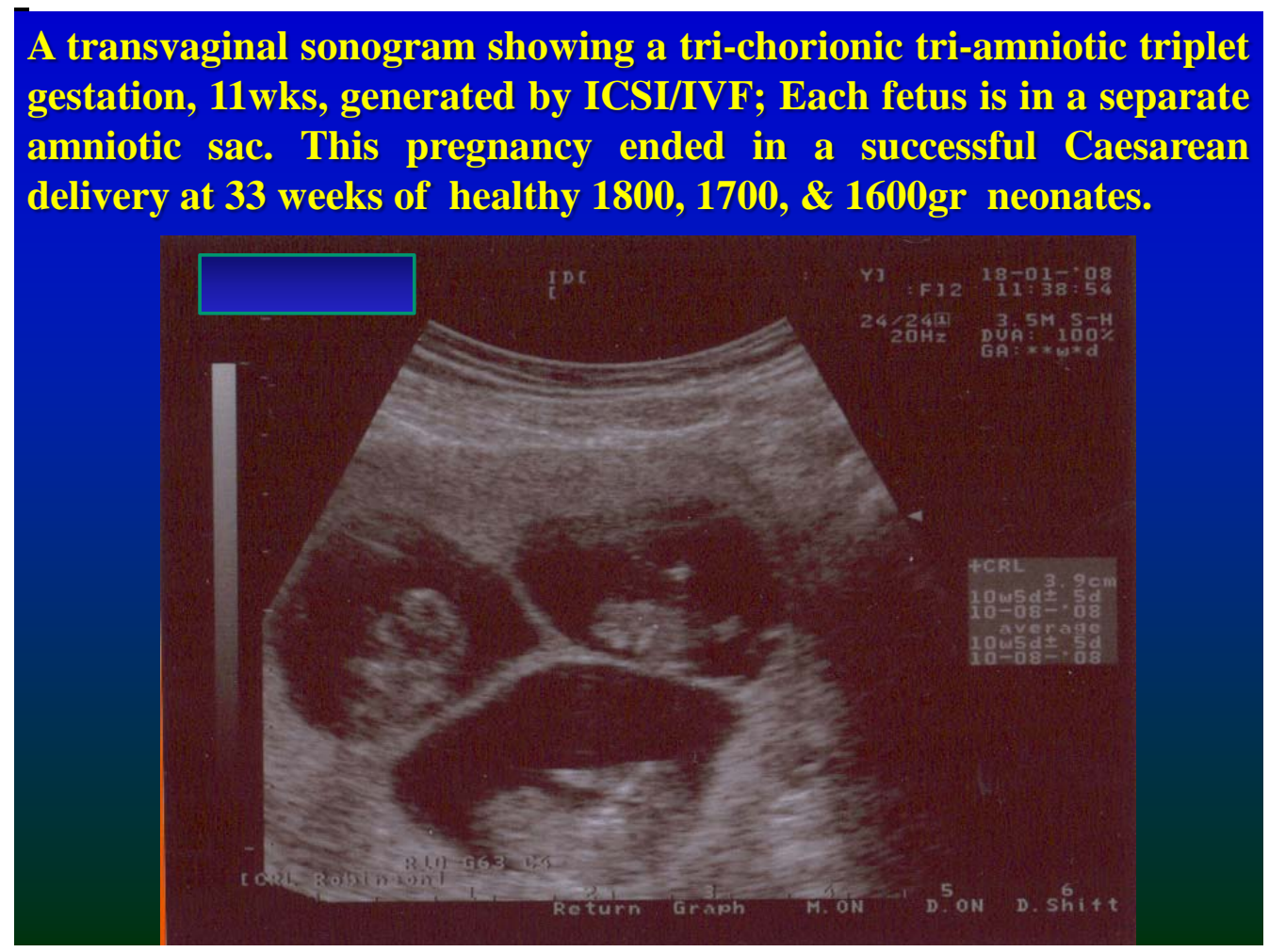

Fig. (2). A transvaginal sonogram showing a tri-chorionic tri-amniotic triplet gestation, 11wks, generated by ICSI/IVF; Each fetus is in a separate amniotic sac. This pregnancy ended in a successful Caesarean delivery at 33 weeks of healthy $1800,1700, \& 1600 \mathrm{gr}$ neonates. 
sels. The BCTA triplets have a birth weight of at least $500 \mathrm{~g}$ less than their TCTA counterparts; although the cause for this observation is unclear, it may be attributed to early spontaneous delivery of BCTA triplets who are complicated with twin to twin transfusion (TTS) and twin reversed arterial perfusion (TRAP) syndromes [3]. The bichorionic triamniotic triplets with discordant weights are usually delivered at least two weeks earlier than those of the TCTA pregnancies [3].

The relative risk of cerebral palsy is 23 folds higher in triplets compared to singletons [26].

There is a paramount importance to determine the type of placentation and chorionicity in all cases of triplet gestation and delivery.

The neonatal morbidity in terms of respiratory distress syndrome, anemia, and intraventricular hemorrhage were higher in bi-chorionic tri-amniotic gestations compared with tri-chorionic tri-amniotic triplets. The premature rupture of the membranes and twin-twin transfusion syndrome were independent risk factors for perinatal death [3].

Monochorionic placentation is associated with a higher risk of intrauterine demise, neonatal death, and complications of prematurity, especially neurological handicaps, compared with bichorionic twins [3]. It is conceivable, therefore, that in triplet pregnancies, the presence of monochorionic fetuses may jeopardize the perinatal outcome. In conclusion, bichorionic triplets are common in spontaneously conceived triplets; the bichorionicity accounts for prematurity and low birth weight with adverse perinatal outcome [3].

Occurrence of chronic TTTS increases significantly the incidence of intrauterine and neonatal death with long term survival of less than $50 \%$ [3].

Triplets had a significantly lower mean average birth weight than twins $(1,596$ vs. 2,317 gram, $P<0.018)$ and lower gestational age at delivery $(32.9 \mathrm{vs} .35 .9$ weeks; $P<$ $0.03)$. Preterm labour occurred significantly more often in triplet than in twin gestations $(80.5 \% v s .41 .5 \%)$ as did preterm delivery $(87.8 \%$ vs. $46.2 \%)$. Triplets required a longer hospital stay than did twins $(25 v s .9$ days; $\boldsymbol{P}<\mathbf{0 . 0 4})$. Low Apgar scores [ $<7$ at 5 minutes] was significantly more frequent in triplet as compared with twin gestation (17vs. 6\%; $P<0.0015)$ [9].

In summary, triplet pregnancies are complicated by a significantly higher incidence of preterm labor and delivery, growth retardation, discordance, and more NICU admissions than are twins. The triplets have an increased risk of several neonatal complications including neonatal death, respiratory distress syndrome, intraventricular hemorrhage, hyperbilirubinaemia, and the need of blood transfusions [9].

\section{NEONATAL OUTCOMES}

Neonatal outcomes of triplets were found to be better in older mothers. Mean gestational age at delivery of mothers aged 40 or older was 33.9 weeks, compared with 32.5 weeks for mothers aged 35 to 39 and 32 weeks for mothers aged 25 to 29. Similarly, the mean total triplet birth weight for mothers aged 40 and older was $5558.5 \mathrm{~g}$, compared with $5153.4 \mathrm{~g}$ for mothers aged 35 to 38 and $4951.1 \mathrm{~g}$ for mothers aged 25 to 29 [12].
Three maternal pregravid characteristics are associated with improved outcome of triplets: multiparous, older, and taller mothers $(>165 \mathrm{~cm})$ do better than nulliparous women, younger than 35 years and shorter patients, with less fetal growth restriction at the gestational age of 27-32 weeks [6].

Parturients older than 40 years had only approximately one third of premature deliveries at less than 28 weeks vs. mothers aged 25 to 29 (2.3\% vs. 6.4\%); mothers older than age 40 gave birth to significantly heavier triplets (all the three fetuses first, second, \& third) $v s$. mothers aged 25 to 29 ( $P=0.16$ for the first fetus ; 0.01 for the second; and 0.03 for the third fetus); total triplet birth weight was significantly higher for mothers older than age 40 compared with mothers aged 25 to $29(P=0.01)$; and births less than $1000 \mathrm{~g}$ were $35 \%$ less frequent in mothers older than age 40 compared with mothers aged 25 to 29 ( $4.5 \%$ vs.7\%), whereas births more that $2.5 \mathrm{~kg}$ accounted for $9.5 \%$ of births in mothers older than age 40 compared with $5.5 \%$ in mothers aged 25 to $29(P=0.005)[12]$.

The likelihood of neonatal, perinatal, and infant mortality was significantly higher among younger mothers. For each unit decrease in maternal age, the odds of stillbirth, neonatal, perinatal, and infant death increased by $9 \%, 12 \%, 13 \%$, and $12 \%$, respectively in a dose-dependent fashion $(P<0.0001)$ [12].

As maternal age advanced from less than twenty to forty years or more, the number of triplet sets with total birth weights of more than $5000 \mathrm{~g}$ increased and the numbers of sets with total birth weights of less than $3000 \mathrm{~g}$ decreased [12].

Zhang et al. [13] analyzed perinatal outcomes in women aged 40 and older (using the same National Center for Health Statistics data set) and found that in contrast to the pattern seen in singleton births, twins born to older women are not at greater risk than twins born to younger women and that triplets born to older women actually fare better than triplets born to younger women. They also concluded that the incidence of very preterm births ( $<32$ weeks), very low birth weight $(<1500 \mathrm{~g})$, perinatal death, and infant death - all progressively declined with advancing maternal age [12].

Retrospective review on the effect of antenatal corticosteroids in triplet pregnancies showed that there was no adverse effect of steroid exposure on weight or head circumference at birth [27].

Overall, short-term and long-term outcomes of triplets do not differ significantly from gestational-matched singletons and twins [7].

Mothers taller than $165 \mathrm{~cm}$ gave birth to significantly heavier neonates than shorter parturients delivered of triplets [1]. Individual and mean total triplet neonatal weights were positively correlated to maternal height [1]. There was no significant correlation between preconception maternal BMI and triplet neonatal weight and week of delivery, NICU hospitalization or any other parameter [1]. The mean weight of neonates in each triplet gestation correlated positively with maternal height [1].

The taller patient $(>165 \mathrm{~cm})$ may be at a significantly lower risk of very low birth weight neonates and very premature delivery as compared to the shorter patient $(<165 \mathrm{~cm})$. 
Therefore, the factor of maternal height should probably be taken into consideration in multiple gestation pregnancy consultations. Shorter mothers should never receive more than two embryos transferred in IVF programs to reduce the risk of triplets almost completely [1].

The mean weight of the neonates in each triplet gestation correlated positively with maternal height [1].

Neonatal Intensive Care Unit (NICU) hospitalization length correlated negatively with maternal height [1].

Perinatal death including intrauterine and neonatal death was found to be more frequent in BCTA than in TCTA triplets [3]. BCTA pregnancies were delivered 2 weeks earlier than TCTA pregnancies $(\boldsymbol{P}<\boldsymbol{0 . 0 1})$, the birth weight of growth-restricted infants was comparable in the two groups. The perinatal mortality rates in BCTA pregnancies were higher than in the TCTA pregnancies $(\boldsymbol{P}<\boldsymbol{0} .05)$ [3].

In multiple pregnancies, preterm delivery is considered to be the most important predictor of neonatal outcome [3].

The overall perinatal mortality rate was 151.5 per 1000 total births [3]. The perinatal loss rate was attributed largely to low birth weight that resulted from preterm delivery because only two infants who were delivered after 29 weeks died in the neonatal period [3]. This is consistent with the observation that perinatal mortality rates for multiple pregnancies increased with increasing prematurity [3].

Neonatal deaths occurred significantly more in triplets than in twins (26 vs. 8.5\%; $\boldsymbol{P}<\mathbf{0 . 0 0 0 1 ) ~ [ 9 ] . ~}$

Apgar score $<7$ at 5 min was significantly more prevalent as compared to twin gestation $(17 v s .6 \% ; \boldsymbol{P}<\boldsymbol{0 . 0 0 1 5})$. Triplets required longer hospital stay than did twins (25 vs. 9 days; $\boldsymbol{P}=\mathbf{0 . 0 4}$ ). There were no significant differences between the groups in number of admissions to the NICU. Neonatal deaths occurred more frequently in triplet than in twin gestations (26\% vs. 8.5\%; $\boldsymbol{P}=\mathbf{0 . 0 0 0 1})$ [9].

There was also a significant difference in the perinatal mortality rate in triplets as compared to twin gestations (260 vs. 85 per 1,000$)$ [9].

Multiple pregnancies are associated with a 7-fold higher rate of neonatal mortality in twins and more than 20-fold higher rate in triplets and higher-order multiples as compared to singletons [10].

There was a three- to six fold higher incidence of cerebral palsy in twins compared with singletons and more than a tenfold higher incidence of cerebral palsy in triplets [10].

Respiratory problems affect most of the infants and include respiratory distress syndrome and apnea of prematurity in the short term and bronchopulmonary dysplasia (BPD) or chronic lung disease in the long term [5].

Neurologic problems characteristic of VLBW infants include intraventricular hemorrhage (IVH) and periventricular leukomalacia. Periventricular leukomalacia is seen less often (4\%-8\% in most series), with peak incidence at approximately 28 to 29 weeks' gestation, in contrast with the incidence of IVH, which seems to correlate inversely with gestational age. Both of these conditions predispose to longterm neurodevelopmental impairment that may include cerebral palsy, mental retardation, and visual, hearing, and be- havioral problems. Sepsis-vertically transmitted and nosocomial infection-is of particular concern in the immunocompromised extremely LBW infant; it affects $20 \%$ to $50 \%$ of these infants, $10 \%$ to $20 \%$ of whom may succumb. Other major morbidities commonly seen in these infants include feeding difficulties, necrotizing enterocolitis, patent ductus arteriosus, and prematurity associated retinopathy [5].

The relative risk for VLBW in twins and triplets as compared to singletons was found to be 9.6 and 32.7, respectively, and for infant mortality - 6.6 and 19.4, respectively [5].

Perinatal mortality is 5-fold higher in twins and 12-fold higher for triplets, as compared to singletons [5].

Respiratory distress syndrome is also significantly more prevalent in twins (70\%; OR 1.58, 95\% CI 1.32-1.89) and triplets (75\%; OR 2.51, 95\% CI 1.87-3.37) when compared to singletons, despite the higher exposure of multiple pregnancies to antenatal steroids [5].

On the other hand, no significant differences were found between the groups regarding the incidence of the major adverse outcomes - chronic lung disease, adverse neurologic findings (severe IVH, periventricular leukomalacia, or ventricular dilatation), or death [5].

In a retrospective review of all the triplets born during a 7 years period, comparisons of the neonatal outcome of the first triplet neonate, to the second, and the third triplet, has shown that the mean birth weight of the first neonate was significantly higher than that of the second and the third delivered triplets. Other measures such as the incidence of RDS, use of surfactant and intubation requirement were lower among the first newborn, but the differences were not statistically significant [28].

The incidence of periventricular leukomalacia, intraventricular hemorrhage, gastroesophgeal reflux, sepsis, and PDA were not statistically different between the three newborn triplets, most probably due to the cesarean mode of delivery all the triplets in that study [28]. The use of IVF was not found to be associated with a significant increased risk of poor outcome, IVH or RDS in triplets versus twins or singletons. The neonatal outcome was similar for ART/IVF and non-ART/IVF generated triplets [28], when the most important determinant of the neonatal outcome was the gestational age [28].

\section{FETAL AND NEONATAL TREATMENT}

Intensive and aggressive modern perinatal care in highrisk pregnancies may possibly benefit the triplets' outcome [5]. Perhaps, in keeping with the recent recommendation in twins, laser ablation may also be a better therapeutic option in triplets with the TTS [3].

\section{PREVENTION, MONITORING, AND TREATMENT}

It is well known that singletons fare better than twins, and twins fare better than triplets. It is also unequivocally accepted that prevention of a triplet pregnancy is better than any "treatment" when such multiples are generated by infertility treatment [6].

The public health implications of high-order multiple pregnancies require prevention and treatment. Prevention 
refers to endeavors attempted towards the elimination of the iatrogenic source of "surplus successes" of infertility treatment, whereas treatment refers to multifetal pregnancy reduction (MFPR) [6].

Two-embryo transfer in ART/IVF in cycles of infertile patients with a good prognosis for conception has been recommended as a way to maintain a high implantation rate while decreasing the number of high-order multiple gestations and at the same time keeping an equivalent overall live birth rate compared with the rate when three or more embryos were transferred [4].

A more intensive surveillance is warranted to promptly diagnose and intervene appropriately for pregnancy-related hypertensive conditions in multifetal gestations [2].

Emphasis must be placed on adequate antenatal care; bed rest, prophylactic use of tocolytics in an attempt to prevent preterm labor, well-equipped neonatal intensive care units [NICU] and specialized care, beginning in early gestation, can have a significant impact on the outcome of mothers and neonates [9].

The recommendation of avoiding the transfer of more than two embryos, especially in women shorter than $165 \mathrm{~cm}$. should be emphasized [1].

By multifetal pregnancy reduction to twins, the rate of triplet deliveries will be decreased although the rate of triplet pregnancies won't be changed [29]. The latter goal should be optimally achieved by restricting the number of embrya transferred to one or two, except in rare and exceptional cases.

\section{MODES OF DELIVERY}

Women aged 40 or over have a higher risk of operative delivery (cesarean, forceps, and vacuum deliveries: 61\%) compared with younger nulliparous women $(35 \%)$. The increase in the rates of operative deliveries despite lower birth weight and gestational age may be explained largely by the higher rates of other pregnancy complications [12].

Effect of mode of delivery on neonatal outcome in triplet vs. twin pregnancies: Several authors have recommended cesarean section as the preferred mode of delivery in triplet gestation. In the study of Ziadeh et al. [9] there was a higher neonatal mortality in the cesarean delivered group as compared to the vaginal route of delivery (30vs. 22\%), which did not confirm a lower mortality or morbidity rate generated by cesarean section, in keeping with other studies [9].

Delivery by caesarean section is much more common in triplets $(89 \%)$ as compared to twins $(65 \%)$ or singleton gestations (22\%) [5].

Although the second twin had an increased risk of malpresentation, the mode of delivery did not significantly influence the outcome [5].

In 1989 Lipitz et al. reported a higher rate of respiratory distress and lower Apgar scores in the vaginally delivered third triplets in comparison with the cesarean mode of delivery for the third triplet [30]. On the other hand, Weissman et al. reported higher perinatal mortality of the second- and third-born neonates despite the cesarean section [31].
A retrospective study on 57 triplets from Albrecht et al. found no difference in the 5-minute Apgar scores, arterial cord $\mathrm{pH}$, or the rate of neonatal complications as a function of birth order [32], suggesting that cesarean section may prevent the neonatal morbidity of the third -born infants [30]. Another retrospective review of 100 triplets' gestations [33] did not find a significant difference in pregnancy outcome with birth order.

\section{SUMMARY}

Although many publications attempted to describe the higher risks and poorer neonatal outcome of triplets' gestations, as compared to twins and singletons, there is still no unequivocally accepted consensus as to the best mode of management. However, there is no doubt that the best treatment is "prevention". It is possible to prevent more than $80 \%$ of triplets by transferring not more than two embryos in ART cycles and by abstaining from intrauterine insemination and intercourse in ovulation-inducing drugs cycles when more than three preovulatory follicles are visualized by transvaginal ultrasound.

A recent survey [34], of 13 studies has found that eight of the 13 studies found most infertile couples to be in favor of multiple birth. In this survey almost none of the infertile couples elected single embryo transfer, about three quarters of the infertile couples required two embrya transferred, and almost one quarter elected to have three embrya transferred. Most couples perceived that single embryo transfer would reduce the chances of having a successful pregnancy, therefore elected to take the risk of multiple gestations for increasing the chance of a "successful" pregnancy. The most important argument for the majority of the infertile couples undergoing ART was the desire to increase the chance of pregnancy and the desire to avoid further treatment. Therefore, the expectation for complete eradication of triplet gestation may not be realistic in the near future...

Whereas the indication for MFPR of triplets to twins is logical, the evidence is still in the order of level II-III and not level-I evidence. Although most care providers would recommend on MFPR of triplets to twins, the procedure is not risk-free. It is recommended by many obstetricians, at present, that triplet gestation undergo iatrogenic fetal reduction to twins in the second trimester, after waiting for spontaneous reductions ("vanished twin" phenomenon) to occur [35], usually before the second trimester. However, in cases of triplet gestation where the couple, in spite of the medical recommendation for fetal reduction, is reluctant to undergo this procedure for moral or religious reasons or due to the fear of losing a long awaited pregnancy, sometimes after longstanding infertility and expensive infertility treatments, the information obtained our study [1], supported by those of Blickstein et al. $(6,11)$ may be helpful. The taller patient $(>165 \mathrm{~cm})$ may be at a significantly lower risk of very low birth weight neonates and very premature delivery as compared to the shorter patient $(<165 \mathrm{~cm})$.

Therefore, the factor of maternal height may be also taken into consideration in multiple gestation pregnancy consultations, since taller mothers deliver heavier babies. In addition, smaller mothers should never receive more than two embryos in IVF programs to reduce the risk of triplets almost completely. 


\section{REFERENCES}

[1] Blumenfeld Z, Lowenstein L, Brook O, Gonen R, Ophir E, Samueloff A. Does maternal height affect triplets' birth weight? Med Sci Monit 2005; 11: CR1-CR4.

[2] Day MC, Barton JR, O'Brien JM, Istwan NB, Sibai BM. The effect of fetal number on the development of hypertensive conditions of pregnancy. Obstet Gynecol 2005; 106: 927-931.

[3] Adegbite AL, Ward SB, Bajoria R. Perinatal outcome of spontaneously conceived triplet pregnancies in relation to chorionicity. Am J Obstet Gynecol 2005; 193: 1463-1471.

[4] Cunningham FG, Leveno KJ, Bloom SL, Hauth JC, Gilstrap III L, Wnstorm KD. Williams Obstetrics $22^{\text {nd }}$ ed, USA: McGraw Hill 2005; 912-922.

[5] Shinwell ES. Neonatal morbidity of very low birth weight infants from multiple pregnancies. Obstet Gynecol Clin N Am 2005; 32: 29-38.

[6] Blickstein I, Rhea DJ, Keith LG. The likelihood of adverse outcomes in triplet pregnancies estimated by pregravid maternal characteristics. Fertil Steril 2004; 81: 1079-1082.

[7] Dickey RP, Taylor SN, Lu PY, et al. Spontaneous reduction of multiple pregnancies: Incidence and effect of outcome. Am J Obstet Gynecol 2002; 186: 77-83.

[8] Andersen AN, Goossens V, Ferraretti AP, et al. European IVFmonitoring (EIM) Consortium, European Society of Human Reproduction and Embryology (ESHRE). Assisted reproductive technology in Europe, 2004: results generated from European registers by ESHRE. Hum Reprod 2008; 23(4): 756-71.

[9] Ziadeh SM. The outcome of triplet versus twin pregnancies. Gynecol Obstet Invest 2000; 50: 96-99.

[10] Baor L, Blickstein I. En route to an "instant family": Psychosocial considerations. Obstet Gynecol Clin N Am 2005; 32: 127-139.

[11] Blickstein I, Keith LG. Aging, twinning, and perinatal outcomes Letter to the editor. Fertil Steril 2003; 79: 661-662.

[12] Oleszczuk JJ, Keith LG, Oleszczuk AK. The paradox of old maternal age in multiple pregnancies. Obstet Gynecol Clin N Am 2005; 32: 69-80.

[13] Zhang J, Meikle S, Grainger DA, Trumble A. Multifetal pregnancy in older women and perinatal outcomes. Fertil Steril 2002; 78: 562568

[14] Dickinson RL. Human sexual anatomy. Baltimore: Williams \& Wilkins 1949; 20-22.

[15] Langlois LP. The size of the normal uterus. J Reprod Med 1970; 4: 220-228.

[16] Elster AD, Bleyl JL, Craven TE. Birth weight standards for triplets under modern obstetric care in the United States, 1984-1989. Obstet Gynecol 1991; 77: 387-393.

[17] McKinney M, Downey J, Timor-Tritsch I. The psychological effects of multifetal pregnancy reduction. Fertil Steril 1995; 64: 5161

[18] Ben-Harush A, Kaplan B, Ben-Rafael Z, Feldberg D. Embryo transfer and multifetal pregnancies - Different aspects of the num- ber of embryos being returned in IVF. Harefuah 2001; 140: 634638.

[19] Yaron Y, Bryan-Greenwood PK, Dave N, et al. Multifetal pregnancy reductions of triplets to twins: Comparison with nonreduced triplets and twins. Am J Obstet Gynecol 1999; 180: 1268-1271.

[20] American College of Obstetricians and Gynecologists: Special problems of multiple gestation. Educational Bulletin 253, Washington DC, November 1998.

[21] Leondires MP, Ernst SD, Miller BT, Scott RT. Triplets: Outcomes of expectant management versus multifetal reduction for $127 \mathrm{preg}$ nancies. Am J Obstet Gynecol 2000; 183: 454-459.

[22] Steinkampf MP. Effect of spontaneous pregnancy reduction on obstetric outcome. J Reprod Med 2005; 50: 603-606.

[23] Lipitz S, Reichman B, Uval J, et al. A prospective comparison of the outcome of triplet pregnancies managed expectantly or by multifetal reduction to twins; Am J Obstet Gynecol 1994; 170: 874879.

[24] Melgar C, Rosenfeld DL, Rawlinson K, Greenberg M. Perinatal outcome after multifetal reduction to twins compared with nonreduced multiple gestations. Obstet Gynecol 1991; 78: 763-767.

[25] Schinzel AA, Smith DW, Miller JR. Monozygotic twinning and structural defects. J Pediatr 1979; 95: 951

[26] Blickstein I. Cerebral palsy and multiple pregnancy. Harefuah 1999; 136: 885-889.

[27] D'Amore A, Ahuluwalia J, Cheema I, Prentice A, Keptoge S, Kelsall W. The effect of antenatal corticosteroids on fetal growth, survival, and neurodevelopmental outcome in triplet pregnancies. Am J Perinatol 2004; 21: 1-8.

[28] Ballabh P, Kumari J, AlKouatly HB, et al. Neonatal outcome of triplet versus twin and singleton pregnancies: A matched case control study. Eur J Obstet Gynecol Reprod Biol 2003; 107: 28-36.

[29] Blickstein I, Baor L. Trends in Multiple Births in Israel. Harefuah 2004; 143: 794-798

[30] Lipitz S, Reichman B, Paret G, et al. The improving outcome of triplet pregnancies. Am J Obstet Gynecol 1989; 161(5): 1279-84.

[31] Weissman A, Yoffe N, Jakobi P, Brandes JM, Paldi E, Blazer S Management of triplet pregnancies in the 1980s--are we doing better? Am J Perinatol 1991; 8(5): 333

[32] Albrecht JL, Tomich PG. The maternal and neonatal outcome of triplet gestations. Am J Obstet Gynecol 1996; 174: 1551-1556.

[33] Devine PC, Malone FD, Athanassiou A, Harvey-Wilkes K D'Alton ME. Maternal and neonatal outcome of 100 consecutive triplet pregnancies. Am J Perinatol 2001; 18: 225-235.

[34] Glazebrook C, Sheard C, Winstanley L, Cox S, Ndukwe G. Attitudes of infertile couples to a multiple birth: a review of the literature and results from a survey. Curr Women's Health Rev 2007; 3 : 43-48.

[35] Blumenfeld Z, Dirnfeld M, Abramovici H, Amit A, Bronshtein M, Brandes JM. Spontaneous fetal reduction in multiple gestations assessed by transvaginal ultrasound. Br J Obstet Gynaecol 1992; 99: 333-7.

(C) Blumenfeld et al.; Licensee Bentham Open.

This is an open access article licensed under the terms of the Creative Commons Attribution Non-Commercial License (http://creativecommons.org/licenses/by-nc/3.0/) which permits unrestricted, non-commercial use, distribution and reproduction in any medium, provided the work is properly cited. 